\title{
A Framework for the Investigation of Biowaste Materials as Potential Adsorbents for Water Treatment
}

\author{
Haliemeh Sweidan $^{1}$, Mohamed Hamouda ${ }^{1}$, Hilal El-Hassan ${ }^{1}$, Munjed Maraqa ${ }^{1}$ \\ ${ }^{1}$ Department of Civil and Environmental Engineering, United Arab Emirates University \\ Al Ain, UAE \\ hsweidan@uaeu.ac.ae; m.hamouda@uaeu.ac.ae; helhassan@uaeu.ac.ae;m.maraqa@uaeu.ac.ae
}

\begin{abstract}
In this paper, a framework for the evaluation of biowaste materials for use in adsorption is presented. The aim is to develop a meticulous and systematic approach to evaluate the use of these materials in water treatment. Biowaste materials have the potential to contribute to the reuse and recycle of wastewater by removing pollutants. The framework included four main stages which were outlined and discussed in details. The framework focused on attributing contaminant removal to specific mechanisms, optimizing the removal/adsorption process, operating a continuous flow adsorption column, and investigating the potential for process scale-up and industrialization/commercialization. The framework was applied to the case study of using eggshells in removing lead from industrial wastewater and results show that the framework adequately outlines the necessary steps. Future studies on biowaste materials and their use as adsorbents can help improve on the framework.
\end{abstract}

Keywords: framework, biowaste, adsorption, water treatment, recycle

\section{Introduction}

Industrial wastewater is often polluted with various substances, including dyes, organic compounds, and heavy metals. The removal of trace heavy metals, in particular, is a serious challenge not only because they dissolve in water and are not easily removed, but also because they pose a serious health risk to humans if congested. For example, copper can cause kidney damage, and intestinal distress [1]. Cadmium and lead are associated with diseases including kidney, cardiovascular, blood, nervous, and bone diseases [2]. Thus, it is important to treat water for heavy metals not only to mitigate serious health risks, but also to recover potentially valuable metals.

The application of natural solutions to solve such serious environmental problems has become the focus of many research projects. Though many other treatment methods are available, such as ion exchange, precipitation, filtration, and coagulation/flocculation [2,3], adsorption with biowaste materials has the important advantage of being cost-effective and environmentally friendly [2]. Biowaste materials are natural by-products of agro-industrial processes. These materials are often disposed of wastefully despite their potential use in various application (e.g. fertilizers, fillers, construction materials, etc.). Thus, the discarded biowaste materials present an environmental problem themselves as they end up occupying landfills, or causing air pollution due to open burning. Many types of biowaste may have properties which qualify them as potential adsorbents. For instance, spent flax meal [4], spent buckwheat hulls [5], date pits [6], rice husk [7, 8], coconut shells [9], apricot pits [10] have been used for the removal of copper, gold, dyes, lanthanum and erbium, and lead, respectively from aqueous solutions. Therefore, their reuse as an adsorbent contributes to both waste management and wastewater treatment solutions.

Certain metrics are applied in order to measure the performance of a biosorbent. The uptake, q, represents the mass of pollutant (in mg) adsorbed per gram of adsorbent. It is calculated as per equation (1), where $c_{i}$ is the initial concentration of the contaminant in $\mathrm{mg} / \mathrm{L}, c_{f}$ is the final concentration of the contaminant in $\mathrm{mg} / \mathrm{L}, V$ is the solution volume in $\mathrm{L}$, and $m$ is the adsorbent mass in grams. Furthermore, the percent removal is calculated according to equation (2).

$$
\begin{gathered}
\mathrm{q}=\frac{\left(\mathrm{c}_{\mathrm{i}}-\mathrm{c}_{\mathrm{f}}\right) \mathrm{V}}{\mathrm{m}_{\mathrm{c}_{i}-\mathrm{c}_{f}}} * 100 \% \\
\mathrm{c}_{\mathrm{i}}
\end{gathered}
$$


The adsorption process is sensitive to many factors and can be physical or chemical, with some adsorbents being more compatible to particular solutes than others. Therefore, this paper aims to develop a framework by which a biowaste can be evaluated for its adsorptive properties for purification of water from various pollutants. The framework sets the guidelines needed to evaluate new biosorbents, which could be scaled-up to industrial applications. An example of using the framework to evaluate eggshells for lead adsorption is also presented.

\section{Framework Development}

Based on the review of relevant literature, a framework was developed to systematically investigate the use biosorbents in water treatment. The framework is shown in Figure 1. First, a biowaste material is selected for investigation as a potential adsorbent. The biowaste should be locally abundant and easy to gather, collect, and store. Dry substances, with low affinity to moisture, are preferred, as there are more adsorption sites to adsorb contaminants in comparison to moist substances, where some sites are occupied by water. Most importantly, the biowaste and its processing should be low cost. After the biowaste is selected, it is washed and ground. These processes help remove impurities and increase the surface area, respectively, thus improving the adsorption. Moreover, some biosorbents may require activation by heat or addition of chemicals to improve their adsorption properties. This decision is informed by the characterization of the biosorbent. For example, BET surface analysis will measure the surface area; a high surface area is desirable in order to improve mass transfer and increase adsorption uptake. Activation methods may be used to increase the surface area of a biosorbent, if the surface area of its natural form was too small and ineffective. Furthermore, particle size distribution analysis gives information on the amount of particles in each size range. It is desirable to have the biosorbent particles as small as possible to increase the surface area without causing operational issues such as clogging in packed columns and phase separation in batch reactors.

Stage 2 entails a mechanistic study that investigates the contribution of each removal mechanism. For instance, lead removal could be due to several processes, including precipitation of the solute, ion exchange of the solute with ions on the biosorbent surface, and adsorption onto the biosorbent surface. Each of these mechanisms should typically be investigated when using a biowaste for lead removal to identify their relative contribution to the total removal. Knowing the dominant removal mechanism will help in identifying the various factors influencing the effectiveness of the treatment process. In general these factors can be classified into those related to: solution physico-chemical characteristics, the biosorbent's characteristics, operational conditions, and reactor design.

Stage 3 describes a study of the adsorption parameters and their optimization. The sensitivity of uptake to each parameter is evaluated using forward elimination by changing one factor at a time, response surface methodology, or 2or 3-level factorial design. The appropriate design, parameters, and their levels are chosen by the investigators based on resources available, project objectives, and relevant literature. Parameters to be studied include adsorbent dose, $\mathrm{pH}$, contact time, temperature, and contaminant concentration. While investigating the $\mathrm{pH}$, it is important to avoid precipitation of the contaminant, as that will give an inaccurate result. An investigator may believe the decrease in contaminant concentration is due to the biosorbent, when, in fact, it could be due to precipitation. Furthermore, isotherm and kinetic studies are carried out. In the former, all parameters are kept constant except for the contaminant concentration. The final concentration, also known as the equilibrium concentration, is measured and the uptake at each concentration is calculated. The data is then fitted to well-known isotherm models such as linear, Langmuir or Freundlich models. This step helps identify the dominant adsorption mechanism and provides the equilibrium data needed to build an adsorption column. On the other hand and in the kinetic study, all parameters are kept constant except for the contact time. This data is then fitted to kinetic models to determine the rate of the reaction and provide input on the design of the adsorption column.

In stage 4, the information collected in stage 3 is used to design an adsorption column. Operational studies are then conducted to determine the conditions for breakthrough concentration and time, clogging issues, and empty bed contact time (EBCT), i.e. the amount of time the water is in contact with the adsorbent. The operating parameters are then iterated and optimized based on the results. In addition to lab-scale adsorption columns, adsorption efficiency of biowaste materials in scaled-up pilot plants is investigated to provide insight into the feasibility of adopting such biowaste in commercial/industrial applications, while also assessing its desorption and regeneration potential. 

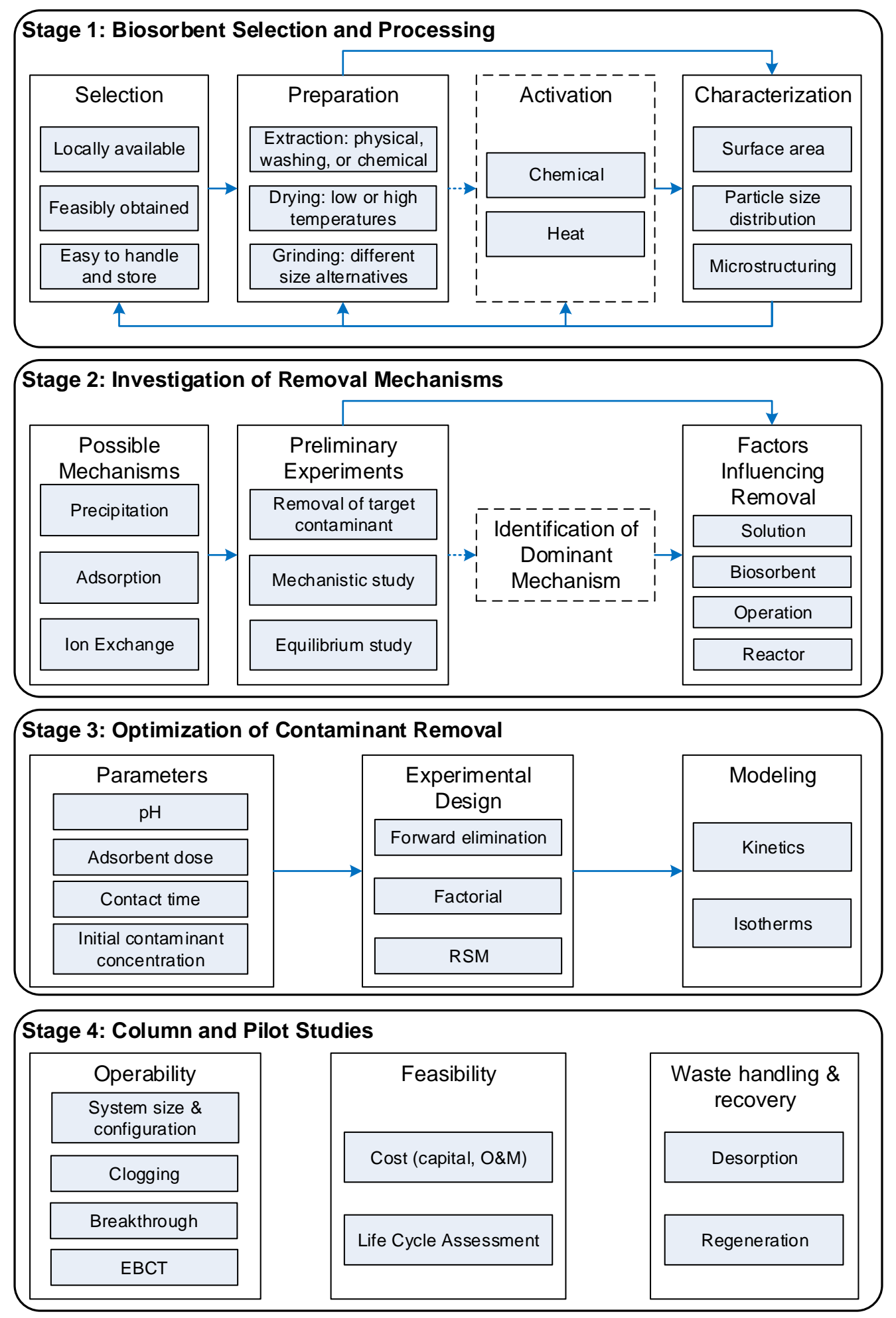

Fig. 1: Framework for investigating biowaste materials as adsorbents.

\section{Framework Demonstration through a Case Study}

The developed framework lays the foundation needed to develop biowaste materials into industrial adsorbents in systematic stages. To evaluate its adequacy, it was applied to eggshells to investigate their potential as a biosorbent to remove lead from industrial wastewater. Eggshells are available in abundance year-round, anywhere in the world. Their chemical 
composition and surface properties suggest an ability to remove various pollutants from water, for it is a dry material, which does not absorb moisture, and its microstructure suggests a high porosity and affinity to many types of solutes [11]. The eggshells used in this case study were sourced from local bakeries and pastry shops. Prior to testing, eggshells samples were washed with deionized water and their membranes were removed. Clean specimens were then dried in an oven at $105^{\circ} \mathrm{C}$ to remove evaporable water and subject to grinding until fine particles were obtained.

A forward elimination method was selected with fine eggshell samples packed into a Perspex lab-scale adsorption column. The investigation comprised the change in uptake with adsorbent dose, lead concentration, $\mathrm{pH}$, and time. Results showed that changing lead concentration and contact time allowed for the collection of equilibrium and kinetic data, which was then used to model adsorption isotherms and kinetics. A continuous flow of lead solution was pumped into the column to simulate wastewater conditions. The lead concentration, bed height, and solution flow rate were varied, and the breakthrough time and concentrations were noted.

\section{Conclusion}

The use of biowaste as potential adsorbents for water treatment promises to contribute to the management of agroindustrial waste and the treatment of water. In alignment with this sustainable initiative, a framework was developed to evaluate biowaste as potential adsorbents for water treatment. The framework presents systematic steps for developing new adsorbents. If successful at the lab and pilot scale, the biowaste-based adsorbents may be industrialized. The proposed framework was employed to assess the use of eggshells for lead adsorption from water. Results confirmed the adequacy of the framework. However, future investigations of other biowaste materials may result in improvements on this framework to make it more generalized and comprehensive.

\section{Acknowledgements}

The authors would also like to express their gratitude to the United Arab Emirates University (UAEU) for financing this project under grants no. 31R150, and 31N153.

\section{References}

[1] F. A. Abu Al-Rub, M. H. El-Naas, I. Ashour and M. Al-Marzouqi, "Biosorption of copper on Chlorella vulgaris from single, binary and ternary metal aqueous solutions," Process Biochemistry, vol. 41, pp. 457-464, 2006.

[2] T. Zhang, Z. Tu, G. Lu, X. Duan, X. Yi, C. Guo, et al., "Removal of heavy metals from acid mine drainage using chicken eggshells in column mode," Journal of Environmental Management, vol. 188, pp. 1-8, 2017.

[3] M. H. El-Naas, S. Al-Zuhair and M. A. Alhaija, "Reduction of COD in refinery wastewater through adsorption on date-pit activated carbon," Journal of Hazardous Materials, vol. 173, pp. 750-757, 2010.

[4] D. Podstawczyk, A. Witek-Krowiak, A. Dawiec and A. Bhatnagar, "Biosorption of copper(II) ions by flax meal: Empirical modeling and process optimization by response surface methodology (RSM) and artificial neural network (ANN) simulation," Ecological Engineering, vol. 83, pp. 364-379, 2015.

[5] P. Yin, M. Xu, R. Qu, H. Chen, X. Liu, J. Zhang, et al., "Uptake of gold (III) from waste gold solution onto biomassbased adsorbents organophosphonic acid functionalized spent buckwheat hulls," Bioresource Technology, vol. 128, pp. 36-43, 2013.

[6] F. Banat, S. Al-Asheh, and L. Al-Makhadmeh, "Evaluation of the use of raw and activated date pits as potential adsorbents for dye containing waters," Process Biochemistry, vol. 39, pp. 193-202, 2003.

[7] Y. Safa and H. N. Bhatti, "Kinetic and thermodynamic modeling for the removal of Direct Red-31 and Direct Orange-26 dyes from aqueous solutions by rice husk," Desalination, vol. 272, pp. 313-322, 2011.

[8] N. S. Awwad, H. M. H. Gad, M. I. Ahmad and H. F. Aly, "Sorption of lanthanum and erbium from aqueous solution by activated carbon prepared from rice husk," Colloids and Surfaces B: Biointerfaces, vol. 81, pp. 593-599, 2010.

[9] M. Sekar, V. Sakthi, and S. Rengaraj, "Kinetics and equilibrium adsorption study of lead (II) onto activated carbon prepared from coconut shell," Journal of Colloid and Interface Science, vol. 279, pp. 307-313, 2004.

[10] M. Rashed, "Fruit stones from industrial waste for the removal of lead ions from polluted water," Environmental monitoring and assessment, vol. 119, pp. 31-41, 2006.

[11] W. Tsai, J. Yang, C. Lai, Y. Cheng, C. Lin, and C. Yeh, "Characterization and adsorption properties of eggshells and eggshell membrane," Bioresource Technology, vol. 97, pp. 488-493, 2006. 